\title{
ANTARES constrains a blazar origin of two IceCube PeV neutrino events ${ }^{\star}$
}

The ANTARES Collaboration: S. Adrián-Martínez ${ }^{1}$, A. Albert ${ }^{2}$, M. André ${ }^{3}$, G. Anton ${ }^{5}$, M. Ardid ${ }^{1}$, J.-J. Aubert ${ }^{6}$, B. Baret ${ }^{7}$, J. Barrios ${ }^{8}$, S. Basa ${ }^{9}$, V. Bertin ${ }^{6}$, S. Biagi ${ }^{24}$, C. Bogazzi ${ }^{12}$, R. Bormuth ${ }^{12,13}$, M. Bou-Cabo ${ }^{1}$, M. C. Bouwhuis ${ }^{12}$, R. Bruijn ${ }^{12,14}$, J. Brunner ${ }^{6}$, J. Busto ${ }^{6}$, A. Capone ${ }^{15,16}$, L. Caramete ${ }^{17}$, J. Carr ${ }^{6}$, T. Chiarusi ${ }^{10}$, M. Circella ${ }^{18}$, R. Coniglione ${ }^{24}$, H. Costantini ${ }^{6}$, P. Coyle ${ }^{6}$, A. Creusot ${ }^{7}$, G. De Rosa ${ }^{19,20}$, I. Dekeyser ${ }^{21,22}$, A. Deschamps ${ }^{23}$, G. De Bonis ${ }^{15,16}$, C. Distefano ${ }^{24}$, C. Donzaud ${ }^{7,25}$, D. Dornic ${ }^{6}$, Q. Dorosti ${ }^{26}$, D. Drouhin ${ }^{2}$, A. Dumas ${ }^{27}$, T. Eberl ${ }^{5}$, A. Enzenhöfer ${ }^{5}$, S. Escoffier ${ }^{6}$, K. Fehn $^{5}$, I. Felis ${ }^{1}$, P. Fermani ${ }^{15,16}$, F. Folger ${ }^{5}$, L. A. Fusco ${ }^{10,11}$, S. Galatà 7 , P. Gay ${ }^{27}$, S. Geißelsöder ${ }^{5}$, K. Geyer ${ }^{5}$, V. Giordano ${ }^{28}$, A. Gleixner ${ }^{5}$, J. P. Gómez-González ${ }^{8}$, R. Gracia-Ruiz ${ }^{7}$, K. Graf ${ }^{5}$, H. van Haren ${ }^{29}$, A. J. Heijboer ${ }^{12}$, Y. Hello ${ }^{23}$, J. J. Hernández-Rey ${ }^{8}$, A. Herrero ${ }^{1}$, J. Höß1 ${ }^{5}$, J. Hofestädt ${ }^{5}$, C. Hugon ${ }^{4,36}$, C. W James ${ }^{5, \star \star}$, M. de Jong ${ }^{12,13}$, O. Kalekin ${ }^{5}$, U. Katz ${ }^{5}$, D. Kießling ${ }^{5}$, P. Kooijman ${ }^{12,14,30}$, A. Kouchner ${ }^{7}$, V. Kulikovskiy ${ }^{24}$, R. Lahmann ${ }^{5}$, D. Lattuada ${ }^{24}$, D. Lefèvre ${ }^{21,22}$, E. Leonora ${ }^{28,32}$, H. Loehner ${ }^{26}$, S. Loucatos ${ }^{33}$, S. Mangano ${ }^{8}$, M. Marcelin ${ }^{9}$, A. Margiotta ${ }^{10,11}$, J. A. Martínez-Mora ${ }^{1}$, S. Martini ${ }^{21,22}$, A. Mathieu ${ }^{6}$, T. Michael ${ }^{12}$, P. Migliozzi ${ }^{19}$, M. Neff ${ }^{5}$, E. Nezri ${ }^{9}$, D. Palioselitis ${ }^{12}$, G. E. Păvălaşş ${ }^{17}$, C. Perrina ${ }^{15,16}$, P. Piattellii ${ }^{24}$, V. Popa ${ }^{17}$, T. Pradier ${ }^{34}$, C. Racca ${ }^{2}$, G. Riccobene ${ }^{24}$, R. Richter ${ }^{5}$, K. Roensch ${ }^{5}$, A. Rostovtsev ${ }^{35}$, M. Saldaña ${ }^{1}$, D. F. E. Samtleben ${ }^{12,13}$, A. Sánchez-Losa ${ }^{8}$, M. Sanguineti ${ }^{4,36}$, P. Sapienza ${ }^{24}$, J. Schmid ${ }^{5}$, J. Schnabel ${ }^{5}$, S. Schulte ${ }^{12}$, F. Schüssler ${ }^{33}$, T. Seitz ${ }^{5}$, C. Sieger ${ }^{5}$, A. Spies ${ }^{5}$, M. Spurio ${ }^{10,11}$, J. J. M. Steijger ${ }^{12}$, Th. Stolarczyk ${ }^{33}$, M. Taiuti ${ }^{4,36}$, C. Tamburini ${ }^{21,22}$, Y. Tayalati ${ }^{37}$, A. Trovato ${ }^{24}$, M. Tselengidou ${ }^{5}$, C. Tönnis ${ }^{8}$, B. Vallage ${ }^{33}$, C. Vallée ${ }^{6}$, V. Van Elewyck ${ }^{7}$, E. Visser ${ }^{12}$, D. Vivolo ${ }^{19,20}$, S. Wagner ${ }^{5}$, E. de Wolf ${ }^{12,14}$, H. Yepes ${ }^{8}$, J. D. Zornoza ${ }^{8}$, J. Zúñiga ${ }^{8}$,

The TANAMI Collaboration: F. Krauß $\beta^{31,38}$, M. Kadler ${ }^{38, \star \star}$, K. Mannheim ${ }^{38}$, R. Schulz ${ }^{31,38}$, J. Trüstedt ${ }^{31,38}$, J. Wilms ${ }^{31}$, R. Ojha ${ }^{39,40,41}$, E. Ros ${ }^{42,43,44}$, W. Baumgartner ${ }^{39}$, T. Beuchert ${ }^{31,38}$, J. Blanchard ${ }^{45}$, C. Bürkel ${ }^{31,38}$, B. Carpenter ${ }^{41}$, P. G. Edwards ${ }^{46}$, D. Eisenacher Glawion ${ }^{38}$, D. Elsässer ${ }^{38}$, U. Fritsch ${ }^{5}$, N. Gehrels ${ }^{39}$, C. Gräfe ${ }^{31,38}$, C. Großberger ${ }^{47}$, H. Hase ${ }^{48}$, S. Horiuchi ${ }^{49}$, A. Kappes ${ }^{38}$, A. Kreikenbohm ${ }^{31,38}$, I. Kreykenbohm ${ }^{31}$, M. Langejahn ${ }^{31,38}$, K. Leiter ${ }^{31,38}$, E. Litzinger ${ }^{31,38}$, J. E. J. Lovell ${ }^{50}$, C. Müller ${ }^{31,38}$, C. Phillips ${ }^{46}$, C. Plötz ${ }^{48}$, J. Quick ${ }^{51}$, T. Steinbring ${ }^{31,38}$, J. Stevens ${ }^{46}$, D. J. Thompson ${ }^{39}$, and A. K. Tzioumis ${ }^{46}$

(Affiliations can be found after the references)

Received 15 January 2015 / Accepted 23 February 2015

\section{ABSTRACT}

Context. The source(s) of the neutrino excess reported by the IceCube Collaboration is unknown. The TANAMI Collaboration recently reported on the multiwavelength emission of six bright, variable blazars which are positionally coincident with two of the most energetic IceCube events. Objects like these are prime candidates to be the source of the highest-energy cosmic rays, and thus of associated neutrino emission.

Aims. We present an analysis of neutrino emission from the six blazars using observations with the ANTARES neutrino telescope.

Methods. The standard methods of the ANTARES candidate list search are applied to six years of data to search for an excess of muons - and hence their neutrino progenitors - from the directions of the six blazars described by the TANAMI Collaboration, and which are possibly associated with two IceCube events. Monte Carlo simulations of the detector response to both signal and background particle fluxes are used to estimate the sensitivity of this analysis for different possible source neutrino spectra. A maximum-likelihood approach, using the reconstructed energies and arrival directions of through-going muons, is used to identify events with properties consistent with a blazar origin.

Results. Both blazars predicted to be the most neutrino-bright in the TANAMI sample (1653-329 and 1714-336) have a signal flux fitted by the likelihood analysis corresponding to approximately one event. This observation is consistent with the blazar-origin hypothesis of the IceCube event IC 14 for a broad range of blazar spectra, although an atmospheric origin cannot be excluded. No ANTARES events are observed from any of the other four blazars, including the three associated with IceCube event IC20. This excludes at a $90 \%$ confidence level the possibility that this event was produced by these blazars unless the neutrino spectrum is flatter than -2.4 .

Key words. neutrinos - galaxies: active - quasars: general

\footnotetext{
$\star$ Figures 2, 3 and Appendix A are available in electronic form at http://www . aanda.org

$\star \star$ Corresponding authors: C. W James, e-mail: clancy.james@physik.uni-erlangen.de;

M. Kadler, e-mail: kadler@physik.uni-wuerzburg.de
} 


\section{Introduction}

Since the initial report of the observation of two high-energy $(\sim \mathrm{PeV})$ neutrino-induced cascades by the IceCube Collaboration (Aartsen et al. 2013), further observations using the high-energy starting-event (HESE) analysis have revealed an excess of events consistent with an isotropic, flavour-uniform flux of astrophysical neutrinos (IceCube Collaboration 2013; Aartsen et al. 2015, 2014). The small number of excess events ( 37 total, with an estimated background of 15), and directional resolution of typically $10^{\circ}$ or worse for cascades, makes it difficult to resolve potential features of this flux, such as a spectral downturn above PeV energies, a steeper spectral index, and/or a contribution from one or more point-like sources of neutrinos. Consequently, many suggestions for the nature and origin(s) of this flux have been put forward. Of particular note is the suggestion of a point-source near the Galactic Centre producing the observed excess in that region (Razzaque 2013), a hypothesis already constrained by the ANTARES Collaboration (Adrián-Martínez et al. 2014a).

The TANAMI Collaboration has recently reported observations of six bright, variable blazars in positional coincidence with the range of possible arrival directions of the two $\mathrm{PeV}$ IceCube events IC 14 and IC 20 (Krauß et al. 2014) ${ }^{1}$. Using a simple calculation based on the observed $1 \mathrm{keV}$ to $10 \mathrm{GeV}$ photon flux, the authors estimate that $1.9 \pm 0.4$ electron neutrino events at $\mathrm{PeV}$ energies would be expected in 662 days of IceCube data. This estimate compares well with the two observed events IC 14 and IC 20. Even taking this only as an orderof-magnitude indication of the expected event rate, a higherresolution follow-up study of these objects is of great interest. Here, we present such an analysis using six years of data from the ANTARES neutrino telescope.

\section{Target blazars and possible neutrino fluxes}

The six blazars associated with the IC 14 and IC 20 fields by Krauß et al. (2014) are listed in Table 1. All exhibit prominent high-energy photon emission, and all but one are classified as flat-spectrum radio quasars (FSRQs; Véron-Cetty \& Véron 2006). The predictions of the expected number of detected electron neutrino events were made by assuming a neutrino energy $E_{v}=1 \mathrm{PeV}$ and a flavour-uniform flux, with total energy flux equal to that in high-energy photons. Active galactic nuclei (AGN) of all classes have long been proposed as sites of hadronic interaction, and are potential sources of the highest-energy cosmic rays and, hence, neutrinos (Berezinskii \& Smirnov 1975; Hillas 1984; Stecker \& Salamon 1996; Padovani $\&$ Resconi 2014). Predictions for the neutrino flux depend on the nature of the AGN considered, the cosmic-ray composition and flux, and the assumed densities of target hadronic matter and magnetic and photon fields (Szabo \& Protheroe 1994; Mannheim 1995; Waxman \& Bahcall 1999; Atoyan \& Dermer 2001; Kelner et al. 2006; Becker Tjus et al. 2014; Dermer et al. 2014).

The emphasis on the two PeV events (IC 14 and IC 20; see Aartsen et al. 2014, for a full list) comes from the fact that these two highest-energy events have only a negligible probability for an atmospheric origin. While IC 14 and IC 20 are assumed to be $v_{\mathrm{e}}$ charged-current (CC) events, and the most common production mechanism (photo-pion production) produces

\footnotetext{
1 The paper was released before the third PeV event, IC 35 ("Big Bird"), was made public. A search for possible blazar associations with this event is in preparation by the TANAMI Collaboration.
}

a flux which is almost uniform in neutrino flavour at Earth, a flavour-dependent flux is predicted by different initial neutrino production mechanisms (Kistler et al. 2014; Anchordoqui 2015), and/or by invoking new physics during propagation (Beacom et al. 2003, and references therein).

The IceCube observations allow for the possibility of a sub$\mathrm{PeV}$ flux of neutrinos from the sample blazars, in that four other events are positionally associated with the blazar sample (see Table 1). This is also consistent with the prediction of two $v_{\mathrm{e}}$ charged-current (CC) events, since the low flavour-dependence of the IceCube HESE effective area at the highest energies means an equal number of $v_{\mu}$ and $v_{\tau}$ events would be expected from a flavour-uniform flux, but with a lower deposited energy. IceCube data are currently compatible with a flavour-uniform flux above $35 \mathrm{TeV}$ (IceCube Collaboration et al. 2015), but a significant excess or deficit of track-like (mostly $v_{\mu} \mathrm{CC}$ ) events in the cosmic diffuse flux cannot be excluded. Thus while these additional four events do not represent a significant excess above a diffuse background, the possibility that they may originate from the blazars in question should also be tested.

\section{ANTARES candidate list search and expected sensitivity}

ANTARES is an underwater neutrino telescope located in the Mediterranean Sea off the coast of Toulon, at $42^{\circ} 48^{\prime} \mathrm{N}, 6^{\circ} 10^{\prime} \mathrm{E}$ (Ageron et al. 2011). Consisting of an array of photomultiplier tubes, it is designed to record the induced Cherenkov light from the passage of energetic charged particles to infer the interactions of neutrinos.

The ANTARES candidate list search (CLS) methodology is described in Adrián-Martínez et al. (2012), with the latest results using six years of data (1338 days effective livetime) presented in Adrián-Martínez et al. (2014a). The search uses only up-going muons (i.e., those originating from below the horizon), with cuts placed on the fit quality of the muon track reconstruction and the estimated angular error. The long range of relativistic muons in seawater and the Earth's crust extends the effective detection volume to well beyond the physical size of the detector, in contrast with a HESE-like analysis. The six-year sample consists of 5516 events, with an estimated atmospheric muon contamination of $10 \%$, and an estimated median angular resolution of $0.38^{\circ}$. A maximum-likelihood method is then used to estimate the relative contributions of signal and background fluxes, based on both the reconstructed event arrival directions and the fitted number of photon hits (a robust proxy for energy). We note that this method results in a non-integer number of signal events $N_{\text {sig }}$ being estimated, since the signal and background fluxes maximising the likelihood of a given observation can take any normalisation. We also note that it is optimised assuming an $E_{v}^{-2}$ source spectrum, and it is sensitive almost exclusively to muon neutrinos. The ability of the ANTARES CLS to constrain the origin of the IceCube events is therefore dependent on the flavour ratio, which may vary according to the neutrino-production scenarios discussed in Sect. 2. Hereafter, sensitivities and limits will be shown for a uniform flavour ratio, from which results for nonuniform flavour fluxes can readily be derived.

The ability of the ANTARES CLS to probe the PeV-neutrino blazar-origin hypotheses of Krauß et al. (2014) can be seen from Fig. 1, which compares the time-integrated, flavour-averaged exposures of the ANTARES CLS (Adrián-Martínez et al. 2014a; 1338 days, using one third of the effective area to muon neutrinos) at the characteristic declinations of the six blazars considered here, to that of the IceCube HESE analysis, averaged 
Table 1. Basic data on the six blazars studied in this analysis.

\begin{tabular}{llcccccccc}
\hline \hline Source & Cat. Name & $\begin{array}{c}\text { RA } \\
{\left[{ }^{\circ}\right]}\end{array}$ & $\begin{array}{c}\text { Dec } \\
{\left[{ }^{\circ}\right]}\end{array}$ & Class & $z$ & $\begin{array}{c}F_{\gamma} \\
{\left[\mathrm{GeV} \mathrm{cm}^{-2} \mathrm{~s}^{-1}\right]}\end{array}$ & $N_{v_{\mathrm{e}}}$ & IC \\
\hline $0235-618$ & PKS 0235-618 & 39.2218 & -61.6043 & $\mathrm{Q}$ & $0.47^{a}$ & $\left(6.2_{-3.1}^{+3.1}\right) \times 10^{-8}$ & $0.19_{-0.04}^{+0.04}$ & 20,7 \\
$0302-623$ & PKS 0302-623 & 45.9610 & -62.1904 & $\mathrm{Q}$ & $1.35^{a}$ & $\begin{array}{c}\left(2.1_{-0.4}^{+0.4}\right) \times 10^{-8} \\
0.06_{-0.01}^{+0.01}\end{array}$ & 20 & 20 \\
$0308-611$ & PKS 0308-611 & 47.4838 & -60.9775 & $\mathrm{Q}$ & $1.48^{a}$ & $\left(4.7_{-1.8}^{+1.8}\right) \times 10^{-8}$ & $0.14_{-0.05}^{+0.05}$ & 20 \\
\hline $1653-329$ & Swift J1656.3-3302 & 254.0699 & -33.0369 & $\mathrm{Q}$ & $2.40^{b}$ & $\left(2.8_{-0.3}^{+0.3}\right) \times 10^{-7}$ & $0.86_{-0.10}^{+0.10}$ & $14,2,25$ \\
$1714-336$ & TXS 1714-336 & 259.4001 & -33.7024 & $\mathrm{~B} / \mathrm{Q}$ & $?$ & $\left(1.5_{-0.4}^{+0.3}\right) \times 10^{-7}$ & $0.46_{-0.12}^{+0.10}$ & $14,2,25$ \\
$1759-396$ & MRC 1759-396 & 270.6778 & -39.6689 & $\mathrm{Q}$ & $1.32^{c}$ & $\left(7.5_{-1.9}^{+1.9}\right) \times 10^{-8}$ & $0.23_{-0.40}^{+0.50}$ & $14,2,15,25$ \\
\hline
\end{tabular}

Notes. Columns: (1) IAU B1950 name; (2) Common catalog name; (3), (4) J 2000 coordinates; (5) Classification: Q - Flat Spectrum Radio Quasar, B - BL Lac object; (6) Redshift: ${ }^{(a)}$ Healey et al. (2008); ${ }^{(b)}$ Cutri et al. (2003); ${ }^{(c)}$ Massaro et al. (2009); (7) Total high-energy photon flux from Krauß et al. (2014); (8) Estimated number $N_{v_{\mathrm{e}}}$ of $v_{\mathrm{e}}$ events in the IceCube 662-day analysis (IceCube Collaboration 2013); (9) IC gives the IceCube event IDs from Aartsen et al. (2014) with which the blazars are positionally consistent within the angular error range from IceCube Collaboration (2013).
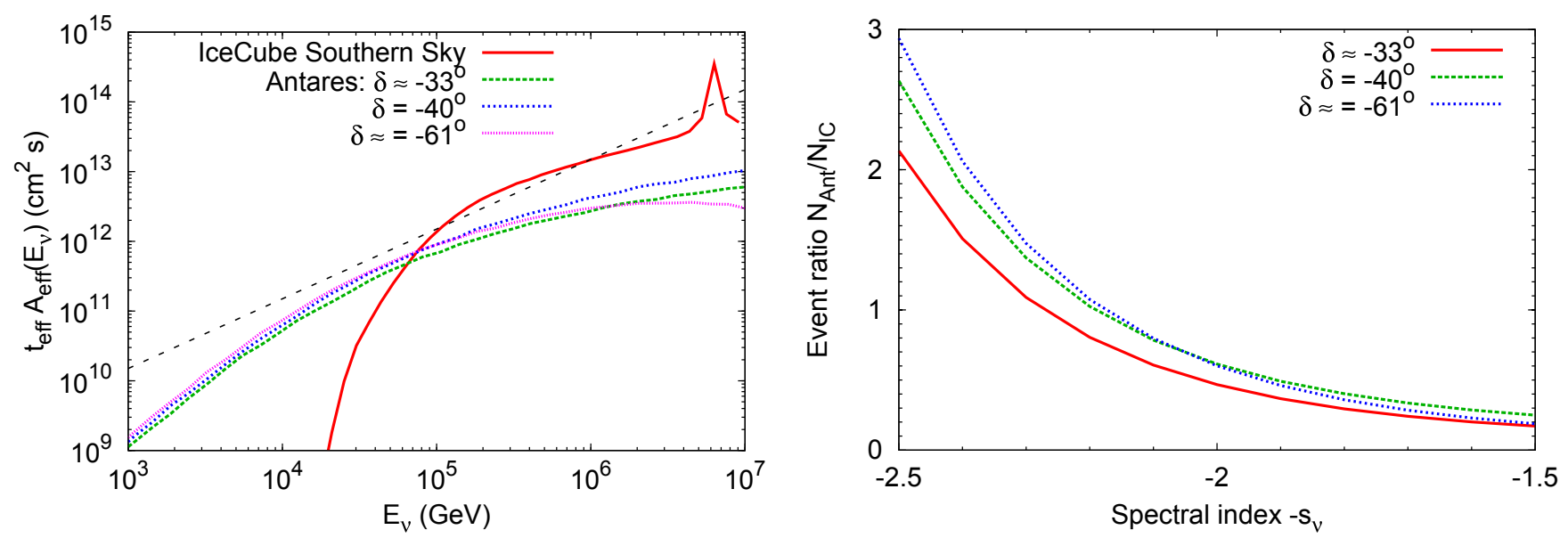

Fig. 1. Left: relative exposures of the ANTARES CLS (Adrián-Martínez et al. 2012) to a flavour-uniform neutrino flux from the characteristic declinations of the six candidate blazars, and the southern-sky-average of the IceCube HESE analysis (Aartsen et al. 2014) (exposures from IceCube Collaboration 2013). The black dashed line, included for reference, is proportional to $E_{\gamma}$. Right: expected number of ANTARES events per detected IceCube event for power-law spectra (Eq. (1)) as a function of the neutrino spectral index $-s_{v}$, calculated using the relative exposures.

over the southern hemisphere (IceCube Collaboration 2013; now updated to 998 days by Aartsen et al. 2014, averaged over all three neutrino flavours). It can be seen that below approximately $100 \mathrm{TeV}$, ANTARES has a greater sensitivity to a neutrino flux from the six blazars at the given southern declinations than the recent IceCube HESE analysis.

The predictions for the number of IceCube-detected PeV neutrino events by Krauß et al. (2014) were based on equating the neutrino flux at $1 \mathrm{PeV}$ to the integrated photon flux between $1 \mathrm{keV}$ and $10 \mathrm{GeV}$. While the expected neutrino-flux shape is highly model-dependent (as was discussed in Sect. 2), the prediction that the total neutrino energy flux $F_{v}\left(\mathrm{GeV} \mathrm{cm} \mathrm{cm}^{-2} \mathrm{~s}^{-1}\right)$ is approximately equal to the total high-energy photon flux $F_{\gamma}$ is relatively robust, at least when attributing this emission to a $100 \%$ hadronic origin. The black-dashed line in Fig. 1 is proportional to neutrino energy $E_{v}$ and normalised to the IceCube exposure at $1 \mathrm{PeV}$, i.e., it is a line of equal sensitivity to a neutrino flux $F_{v}$. For constant $F_{v}$, it is clear that the IceCube HESE analysis is most sensitive to a flux at a few hundred $\mathrm{TeV}$, while the ANTARES CLS is most sensitive near $30 \mathrm{TeV}$.

The range of potential neutrino spectra, $\Phi_{v}\left(E_{v}\right)\left(\mathrm{d} N_{v} / \mathrm{d} E_{v}\right)$, are characterised by generic power-law spectra with spectral index $-s_{v}$ :

$\Phi_{v}\left(E_{v}\right)=\Phi_{0}\left(\frac{E_{v}}{1 \mathrm{GeV}}\right)^{-s_{v}}\left[\mathrm{GeV}^{-1} \mathrm{~cm}^{-2} \mathrm{~s}^{-1}\right]$.
The relative numbers of events expected to be observed by ANTARES compared to IceCube for such spectra are shown in Fig. 1 (right). The required energy in such fluxes to produce a single detectable event in ANTARES is calculated in Appendix A, and plotted in Fig. 2. In the range $-2.5<-s_{v}<$ -1.5 , it is comparable with the total blazar photon flux calculated by Krauß et al. (2014) (see Table 1).

Having established a wide range of plausible neutrino flux scenarios, and the sensitivity of the ANTARES CLS to neutrino fluxes over a broad range of energies, we therefore perform the standard ANTARES CLS for an excess of neutrino emission from the six candidate blazars.

\section{Results and discussion}

The results of the ANTARES analysis of the six blazars are given in Table 2. For four of the six targets, no source-like neutrinos were identified $\left(N_{\text {sig }}=0\right)$, allowing relatively strong upper limits to be placed on an $E_{v}^{-2}$ flux. Blazars $1653-329$ and 1714-336 were each fitted as having approximately one nearby signal-like event, with $N_{\text {sig }}$ of 1.1 and 0.9 respectively ${ }^{2}$. This observation is well within the expected background fluctuations, however,

\footnotetext{
2 The maximum-likelihood procedure estimates $N_{\text {sig }}$ as a continuous variable, as discussed in Sect. 3.
} 
Table 2. ANTARES point-source analysis results.

\begin{tabular}{lccccccc}
\hline \hline Source & $N_{\text {sig }}$ & $p$ & Limit & \multicolumn{4}{c}{$N_{v, \text { IC }}=1,2,3,4$} \\
\hline $0235-618$ & 0 & 1 & 1.3 & -2.4 & -2.1 & -2.0 & -1.9 \\
$0302-623$ & 0 & 1 & 1.3 & -2.4 & -2.1 & -2.0 & -1.9 \\
$0308-611$ & 0 & 1 & 1.3 & -2.4 & -2.1 & -2.0 & -1.9 \\
\hline $1653-329$ & 1.1 & 0.10 & 2.9 & $<-2.5$ & -2.5 & -2.3 & -2.2 \\
$1714-336$ & 0.9 & 0.04 & 3.5 & $<-2.5$ & -2.5 & -2.3 & -2.2 \\
$1759-396$ & 0 & 1 & 1.4 & -2.4 & -2.1 & -2.0 & -1.8 \\
\hline
\end{tabular}

Notes. Columns: (1) IAU B 1950 name; (2) number of fitted signal events; (3) pre-trial $p$-value; (4) $90 \%$ upper limit $\left(10^{-8} \mathrm{GeV}^{-1} \mathrm{~cm}^{-2} \mathrm{~s}^{-1}\right)$ on $\Phi_{0}$ for $-s_{v}=-2.0$; (5): minimum spectral indices $-s_{v}$ consistent at $90 \%$ C.L. with $N_{v, \text { IC }}=1 \ldots 4$ associated IceCube events. Limits assume a flavour-uniform flux.

with pre-trial $p$-values (probability of the likelihood procedure fitting a stronger signal flux to background-only data) of 0.10 and 0.04 , respectively ${ }^{3}$. Nonetheless, it must be noted that these two blazars have the highest predicted neutrino fluxes, and that from Fig. 1 (right), neutrino fluxes with spectral indices between -2.5 and -2.3 producing one IceCube event would be expected to produce between one and two ANTARES events. Therefore, when the calculation of Krauß et al. (2014) is extended to include power-law neutrino spectra, the result of the analysis is consistent with the sample blazars being neutrino sources with fluxes in proportion to their observed high-energy photon flux $\left(F_{\gamma}\right.$ in Table 1), even if the result is also consistent with background.

Limits at a $90 \%$ confidence level (C.L.), $\Phi_{v}^{90}$, on the spectra from Eq. (1) are generated from the ANTARES observations as a function of $s_{v}$ over the approximate predicted range (between 1.5 and 2.5), using the method of Neyman (1937). All are upper limits and are given in Fig. 3 (left). The confidence level is given at $100 \mathrm{TeV}$, because it is both the approximate energy at which the ANTARES and IceCube analyses have equal exposures and where the flux limit is least sensitive to $s_{v}$.

The flux limits correspond to a maximum expected number $N_{v, \text { IC }}^{90}$ of events observed by IceCube; where this number is less than the observed number of events, a blazar origin can be excluded at $90 \%$ C.L. This is shown in Fig. 3 (right). Any given number of IceCube events is therefore only consistent with a blazar origin for neutrino spectral indices flatter than certain value; minimum values of $-s_{v}$ are given for $1-4$ events in Table 2 and should be compared to the possible associations in Table 1. For the IC 14 field for instance, the possibility that blazar 1759-396 could be responsible for three or more associated IceCube events is excluded at $90 \%$ confidence for neutrino spectra steeper than -2.1 . For spectra steeper than -2.4 , we can exclude that $1759-396$ is responsible for any IceCube events. The limits for 1653-329 and 1714-336 are weaker because of a possible physical association with the two signal-like ANTARES events. Regardless of the association, we can rule out the possibility that the cluster IC 14, IC 2, and IC 25 arose from a single considered blazar with a spectrum steeper than -2.4 . For the IC 20 grouping, the non-observation of any event from the three candidate blazars means that the $\delta \approx-61^{\circ}$ limit applies both to the individual blazars, and the group as a whole. Therefore, ANTARES observations can rule out a neutrino spectrum steeper than -2.2 as being responsible for both IC 20 and IC 7, and a neutrino spectrum steeper than -2.4 being responsible for only one of them. That is, if IC 20 does indeed originate

\footnotetext{
3 The correct penalty factor for multiple trials is 61 , including the six blazars considered here, and 55 trials from other analyses using the CLS (Adrián-Martínez et al. 2014a,b).
}

from the three associated TANAMI blazars, the neutrino spectral index must be flatter than -2.4 .

\section{Conclusion}

We have tested the hypothesis of Krauß et al. (2014) that the first two PeV neutrino events observed by IceCube, IC 14 and IC 20, are of blazar origin, by performing a CLS for an excess muon neutrino flux from the six suggested blazars using six years of ANTARES data. We are not able to either confirm or rule out a blazar origin of these events, although constraints have been placed on the range of source spectra which could have produced them, particularly in the case of IC 20. These constraints assume that muon neutrinos constitute one third of the neutrino flux, and strengthen or weaken proportionally with this fraction. While approximately two ANTARES events were fitted as being more signal-like than background-like by the maximumlikelihood analysis, such a result is completely within the expected background fluctuations, with pre-trial $p$-values of $10 \%$ and $4 \%$ for the blazars in question (1653-329 and 1714-336). It is interesting to note that these two blazars were predicted by Krauß et al. (2014) to have the strongest neutrino flux, and that such a result is within expectations for the ANTARES event rate for an $E_{v}^{-2}$ to $E_{v}^{-2.3}$ neutrino spectrum given that IceCube observes two such events, and $E_{v}^{-2.3}$ to $E_{v}^{-2.5}$ for a single event of blazar origin. Given these considerations, the TANAMI candidate blazars should be included in all future analyses.

Acknowledgements. The authors would like to thank A. Kappes for helpful discussions regarding the IceCube analysis. The ANTARES authors acknowledge the financial support of the funding agencies: Centre National de la Recherche Scientifique (CNRS), Commissariat à l'énegie atomique et aux énergies alternatives (CEA), Commission Européenne (FEDER fund and Marie Curie Program), Région Alsace (contrat CPER), Région Provence-Alpes-Côte d'Azur, Département du Var and Ville de La Seyne-sur-Mer, France; Bundesministerium für Bildung und Forschung (BMBF), Germany; Istituto Nazionale di Fisica Nucleare (INFN), Italy; Stichting voor Fundamenteel Onderzoek der Materie (FOM), Nederlandse organisatie voor Wetenschappelijk Onderzoek (NWO), the Netherlands; Council of the President of the Russian Federation for young scientists and leading scientific schools supporting grants, Russia; National Authority for Scientific Research (ANCS), Romania; Ministerio de Ciencia e Innovación (MICINN), Prometeo of Generalitat Valenciana and MultiDark, Spain; Agence de l'Oriental and CNRST, Morocco. We also acknowledge the technical support of Ifremer, AIM and Foselev Marine for the sea operation and the CC-IN2P3 for the computing facilities. The TANAMI authors acknowledge support and partial funding by the Deutsche Forschungsgemeinschaft grant WI 1860-10/1 (TANAMI) and GRK 1147, Deutsches Zentrum für Luft- und Raumfahrt grant 50 OR 1311/50 OR 1303/50 OR 1401, the Spanish MINECO project AYA201238491-C02-01, the Generalitat Valenciana project PROMETEOII/2014/057, the COST MP0905 action "Black Holes in a Violent Universe" and the Helmholtz Alliance for Astroparticle Physics (HAP).

\section{References}

Aartsen, M. G., Abbasi, R., Abdou, Y., et al. 2013, Phys. Rev. Lett., 111, 021103 Aartsen, M. G., Ackermann, M., Adams, J., et al. 2014, Phys. Rev. Lett., 113, 101101

Aartsen, M. G., Ackermann, M., Adams, J., et al. 2015, Phys. Rev. Lett., 91, 022001

Adrián-Martínez, S., Samarai, I. A., Albert, A., et al. 2012, ApJ, 760, 53

Adrián-Martínez, S., Albert, A., André, M., et al. 2014a, ApJ, 786, L5

Adrián-Martínez, S., Albert, A., André, M., et al. 2014b, JCAP, 11, 017

Ageron, M., Aguilar, J. A., Al Samarai, I., et al. 2011, Nucl. Instr. Meth. Phys. Res. A, 656, 11

Anchordoqui, L. A. 2015, Phys. Rev. D, 91, 027301

Atoyan, A., \& Dermer, C. D. 2001, Phys. Rev. Lett., 87, 221102

Beacom, J. F., Bell, N. F., Hooper, D., Pakvasa, S., \& Weiler, T. J. 2003, Phys. Rev. D, 68, 093005

Becker Tjus, J., Eichmann, B., Halzen, F., Kheirandish, A., \& Saba, S. M. 2014, Phys. Rev. D, 89, 123005 
Berezinskii, V. S., \& Smirnov, A. I. 1975, Ap\&SS, 32, 461

Cutri, R. M., Skrutskie, M. F., van Dyk, S., et al. 2003, VizieR Online Data Catalog: II $/ 246$

Dermer, C. D., Murase, K., \& Inoue, Y. 2014, J. High Energy Astrophys., 3, 29

Healey, S. E., Romani, R. W., Cotter, G., et al. 2008, ApJS, 175, 97

Hillas, A. M. 1984, ARA\&A, 22, 425

IceCube Collaboration 2013, Science, 342, 1242856

IceCube Collaboration, Aartsen, M. G., Ackermann, M., et al. 2015, Phys. Rev. Lett., submitted [arXiv: 1502.03376]

Kelner, S. R., Aharonian, F. A., \& Bugayov, V. V. 2006, Phys. Rev. D, 74, 034018

Kistler, M. D., Stanev, T., \& Yüksel, H. 2014, Phys. Rev. D, 90, 123006

Krauß, F., Kadler, M., Mannheim, K., et al. 2014, A\&A, 566, L7

Mannheim, K. 1995, Astropart. Phys., 3, 295

Massaro, E., Giommi, P., Leto, C., et al. 2009, A\&A, 495, 691

Neyman, J. 1937, Roy. Soc. London Phil. Trans. Ser. A, 236, 333

Padovani, P., \& Resconi, E. 2014, MNRAS, 443, 474

Razzaque, S. 2013, Phys. Rev. D, 88, 081302

Stecker, F. W., \& Salamon, M. H. 1996, Space Sci. Rev., 75, 341

Szabo, A. P., \& Protheroe, R. J. 1994, Astropart. Phys., 2, 375

Véron-Cetty, M.-P., \& Véron, P. 2006, A\&A, 455, 773

Waxman, E., \& Bahcall, J. 1999, Phys. Rev. D, 59, 023002

1 Institut d'Investigació per a la Gestió Integrada de les Zones Costaneres (IGIC) - Universitat Politècnica de València. C/ Paranimf 1, 46730 Gandia, Spain

2 GRPHE - Université de Haute Alsace - Institut Universitaire de Technologie de Colmar, 34 rue du Grillenbreit, BP 50568, 68008 Colmar, France

3 Technical University of Catalonia, Laboratory of Applied Bioacoustics, Rambla Exposició, 08800 Vilanova i la Geltrú, Barcelona, Spain

4 INFN-Sezione di Genova, via Dodecaneso 33, 16146 Genova, Italy

5 Friedrich-Alexander-Universität Erlangen-Nürnberg, Erlangen Centre for Astroparticle Physics, Erwin-Rommel-Str. 1, 91058 Erlangen, Germany

${ }^{6}$ CPPM, Aix-Marseille Université, CNRS/IN2P3, 13009 Marseille, France

7 APC, Université Paris Diderot, CNRS/IN2P3, CEA/IRFU, Observatoire de Paris, Sorbonne Paris Cité, 75205 Paris, France

${ }^{8}$ IFIC - Instituto de Física Corpuscular, Edificios Investigación de Paterna, CSIC - Universitat de València, Apdo. de Correos 22085, 46071 Valencia, Spain

9 LAM - Laboratoire d'Astrophysique de Marseille, Pôle de l'Étoile Site de Château-Gombert, rue Frédéric Joliot-Curie 38, 13388 Marseille Cedex 13, France

10 INFN-Sezione di Bologna, viale Berti-Pichat 6/2, 40127 Bologna, Italy

11 Dipartimento di Fisica e Astronomia dell'Università, Viale BertiPichat 6/2, 40127 Bologna, Italy

12 Nikhef, Science Park, 1098 XG Amsterdam, The Netherlands

13 Huygens-Kamerlingh Onnes Laboratorium, Universiteit Leiden, 2300 RA Leiden, The Netherlands

14 Universiteit van Amsterdam, Instituut voor Hoge-Energie Fysica, Science Park 105, 1098 XG Amsterdam, The Netherlands

15 INFN-Sezione di Roma, P.le Aldo Moro 2, 00185 Roma, Italy

16 Dipartimento di Fisica dell'Università La Sapienza, P.le Aldo Moro 2, 00185 Roma, Italy

17 Institute for Space Science, 077125 Bucharest, Măgurele, Romania

18 INFN-Sezione di Bari, via E. Orabona 4, 70126 Bari, Italy

19 INFN-Sezione di Napoli, via Cintia 80126 Napoli, Italy

20 Dipartimento di Fisica dell'Università Federico II di Napoli, via Cintia 80126 Napoli, Italy
21 Mediterranean Institute of Oceanography (MIO), Aix-Marseille University, 13288 Marseille, Cedex 9, France

22 Université du Sud Toulon-Var, CNRS-INSU/IRD UM 110, 83957 La Garde Cedex, France

23 Géoazur, Université Nice Sophia-Antipolis, CNRS/INSU, IRD, Observatoire de la Côte d'Azur, Sophia Antipolis, France

24 INFN-Laboratori Nazionali del Sud (LNS), via S. Sofia 62, 95123 Catania, Italy

25 Univ. Paris-Sud, 91405 Orsay Cedex, France

26 Kernfysisch Versneller Instituut (KVI), University of Groningen, Zernikelaan 25, 9747 AA Groningen, The Netherlands

27 Laboratoire de Physique Corpusculaire, Clermont Université, Université Blaise Pascal, CNRS/IN2P3, BP 10448, 63000 Clermont-Ferrand, France

28 INFN-Sezione di Catania, viale Andrea Doria 6, 95125 Catania, Italy

29 Royal Netherlands Institute for Sea Research (NIOZ), Landsdiep 4, 1797 SZ 't Horntje (Texel), The Netherlands

${ }^{30}$ Universiteit Utrecht, Faculteit Betawetenschappen, Princetonplein 5, 3584 CC Utrecht, The Netherlands

31 Dr. Remeis-Sternwarte and ECAP, Universität Erlangen-Nürnberg, Sternwartstr. 7, 96049 Bamberg, Germany

32 Dipartimento di Fisica ed Astronomia dell'Università, viale Andrea Doria 6, 95125 Catania, Italy

33 Direction des Sciences de la Matière - Institut de recherche sur les lois fondamentales de l'Univers - Service de Physique des Particules, CEA Saclay, 91191 Gif-sur-Yvette Cedex, France

34 IPHC-Institut Pluridisciplinaire Hubert Curien - Université de Strasbourg et CNRS/IN2P3, 23 rue du Loess, BP 28, 67037 Strasbourg Cedex 2, France

35 ITEP - Institute for Theoretical and Experimental Physics, B. Cheremushkinskaya 25, 117218 Moscow, Russia

36 Dipartimento di Fisica dell’Università, via Dodecaneso 33, 16146 Genova, Italy

37 University Mohammed I, Laboratory of Physics of Matter and Radiations, BP 717, 6000 Oujda, Morocco

38 Institut für Theoretische Physik und Astrophysik, Universität Würzburg, Emil-Fischer-Str. 31, 97074 Würzburg, Germany

39 NASA, Goddard Space Flight Center, Greenbelt, MD 20771, USA

${ }^{40}$ University of Maryland, Baltimore County, Baltimore, MD 21250, USA

41 Catholic University of America, Washington, DC 20064, USA

42 Max-Planck-Institut für Radioastronomie, Auf dem Hügel 69, 53121 Bonn, Germany

43 Departament d'Astronomia i Astrofísica, Universitat de València, C/ Dr. Moliner 50, 46100 Burjassot, València, Spain

44 Observatori Astronòmic, Universitat de València, C/ Catedrático José Beltrán No. 2, 46980 Paterna, València, Spain

45 Departamento de Astronomía, Universidad de Concepción, Casilla 160, Chile

46 CSIRO Astronomy and Space Science, ATNF, PO Box 76 Epping, NSW 1710, Australia

47 Max-Planck-Institut für extraterrestrische Physik, Giessenbachstraße 1, 85741 Garching, Germany

48 Bundesamt für Kartographie und Geodäsie, 93444 Bad Kötzting, Germany

49 CSIRO Astronomy and Space Science, Canberra Deep Space Communications Complex, PO Box 1035, Tuggeranong, ACT 2901, Australia

50 School of Mathematics \& Physics, University of Tasmania, Private Bag 37, Hobart, Tasmania 7001, Australia

51 Hartebeesthoek Radio Astronomy Observatory, 1740 Krugersdorp, South Africa 


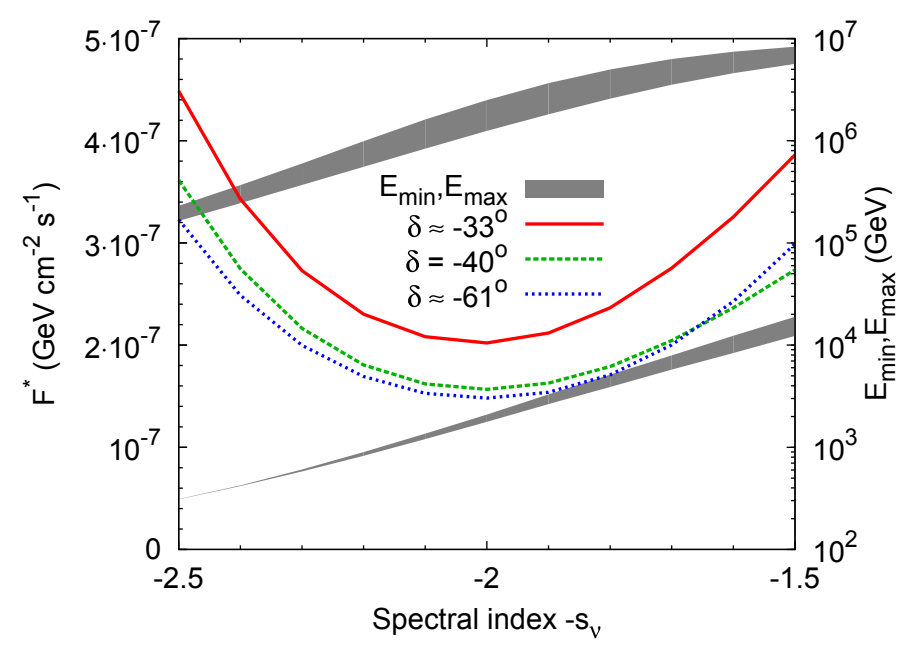

Fig. 2. Neutrino flux $F_{v}^{*}$ required to produce one neutrino event in ANTARES as a function of spectral index $s_{v}$ (Eq. (A.3)). The corresponding energy ranges of integration $E_{\min }$ and $E_{\max }$ (Eq. (A.2)) are shown as lower and upper shaded regions respectively: the shading covers the variation due to declination.
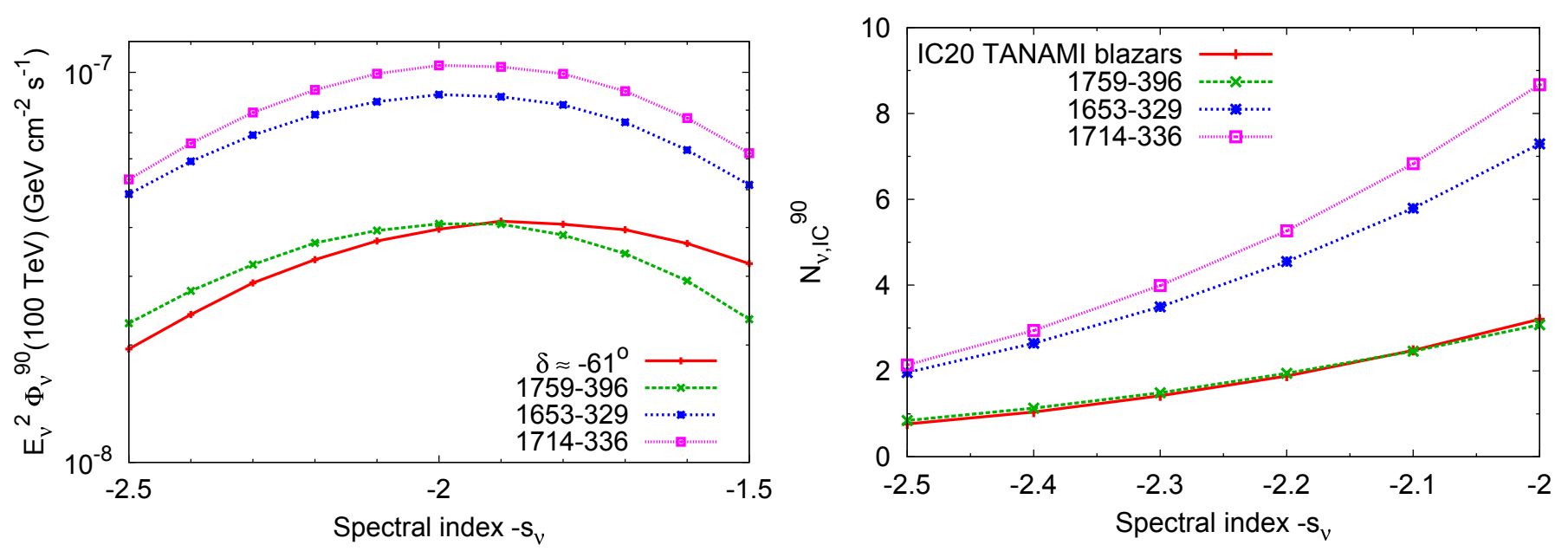

Fig. 3. Left: ANTARES $90 \%$ confidence limits on a flavour-uniform neutrino flux $\left(\Phi_{v} \equiv \Phi_{v_{\mathrm{e}}}+\Phi_{v_{\mu}}+\Phi_{\nu_{\tau}}=3 \Phi_{v_{\mu}}\right)$ from the six blazars as a function of spectral index $s_{v}$ (Eq. (1)). Right: corresponding limits on the expected number of IceCube events of blazar origin, using the exposures shown in Fig. 1 and the limiting fluxes. Since the limits from 0235-618, 0302-623, and 0308-611 are almost identical, and since no events were observed, the limits also apply to the summed flux from all three of these blazars, and hence only one line is shown, and is labelled "IC 20 TANAMI blazars".

\section{Appendix A: Calculation of neutrino energy flux}

For each spectral index $-s_{v}$ and source declination $\delta$, the required neutrino flux $\Phi_{v}^{*}\left(E_{v}, \delta\right)$ expected to produce a single ANTARES event can be found from the expression

$\int_{0}^{\infty} t_{\text {eff }} A_{\text {eff }}\left(E_{v}\right) \Phi_{v}^{*}\left(E_{v}, \delta\right) \mathrm{d} E_{v}=1$,

where $A_{\text {eff }}\left(E_{v}, \delta\right)$ and $t_{\text {eff }}$ are respectively the ANTARES effective area and the observation time. While the total energy in such a flux is infinite, the energy over the sensitive range of ANTARES can be calculated by defining characteristic energies $E_{\min }\left(\delta, s_{v}\right)$ and $E_{\max }\left(\delta, s_{v}\right)$ such that:

$\int_{E_{\min }}^{E_{\max }} t_{\mathrm{eff}} A_{\text {eff }}\left(E_{v}, \delta\right) \Phi_{\nu}^{*}\left(E_{v}, \delta\right) \mathrm{d} E_{v}=0.9$, with 0.05 below $E_{\min }$ and 0.05 above $E_{\max }$. The total neutrino energy flux $F_{v}^{*}\left(\delta, s_{v}\right)$ in the range $E_{\min } \leq E_{v} \leq E_{\max }$ required to produce one event can then be calculated from $\Phi_{v}^{*}\left(E_{v}, \delta\right)$ as:

$F_{v}^{*}\left(\delta, s_{v}\right)=\frac{1}{0.9} \int_{E_{\min }}^{E_{\max }} \Phi_{v}^{*}\left(E_{v}, \delta\right) E_{v} \mathrm{~d} E_{v}\left[\mathrm{GeV} \mathrm{cm}^{-2} \mathrm{~s}^{-1}\right]$

In Fig. $2 F_{v}^{*}\left(\delta, s_{v}\right)$ is plotted along with $E_{\min }$ and $E_{\max }$. 